\title{
Advancement in research on the role of the transient receptor potential vanilloid channel in cerebral ischemic injury (Review)
}

\author{
QIAN XIE* , RONG MA* , HONGYAN LI, JIAN WANG, XIAOQING GUO and HAI CHEN \\ School of Pharmacy and State Key Laboratory of Characteristic Chinese Medicine Resources in Southwest China, \\ Chengdu University of Traditional Chinese Medicine, Chengdu, Sichuan 611137, P.R. China
}

Received June 3, 2020; Accepted May 28, 2021

DOI: $10.3892 /$ etm.2021.10313

\begin{abstract}
Stroke is a common critical disease occurring in middle-aged and elderly individuals, and is characterized by high morbidity, lethality and mortality. As such, it is of great concern to medical professionals. The aim of the present review was to investigate the effects of transient receptor potential vanilloid (TRPV) subtypes during cerebral ischemia in ischemia-reperfusion animal models, oxygen glucose deprivation and in other administration cell models in vitro to explore new avenues for stroke research and clinical treatments. TRPV1, TRPV2 and TRPV4 employ different methodologies by which they confer protection against cerebral ischemic injury. TRPV1 and TRPV4 are likely related to the inhibition of inflammatory reactions, neurotoxicity and cell apoptosis, thus promoting nerve growth and regulation of intracellular calcium ions $\left(\mathrm{Ca}^{2+}\right)$. The mechanisms of neuroprotection of TRPV1 are the JNK pathway, N-methyl-D-aspartate (NMDA) receptor and therapeutic hypothermia. The mechanisms of neuroprotection of TRPV4 are the PI3K/Akt pathways, NMDA receptor and p38 MAPK pathway, amongst others. The mechanisms by which TRPV2 confers its protective effects are predominantly connected with the regulation of nerve growth factor, MAPK and JNK pathways, as well as JNK-dependent pathways. Thus, TRPVs have the potential for improving outcomes associated with cerebral ischemic or reperfusion injuries. The protection conferred by TRPV1 and TRPV4 is closely related to cellular $\mathrm{Ca}^{2+}$ influx, while TRPV2 has a different target and mode of action, possibly due to its expression sites. However, in light of certain contradictory research conclusions, further
\end{abstract}

Correspondence to: Professor Jian Wang, School of Pharmacy and State Key Laboratory of Characteristic Chinese Medicine Resources in Southwest China, Chengdu University of Traditional Chinese Medicine, 1166 Liutai Avenue, Chengdu, Sichuan 611137, P.R. China E-mail: jianwang08@163.com

*Contributed equally

Key words: transient receptor potential vanilloid, cerebral ischemic injury, ischemic stroke, research progress experimentation is required to clarify the mechanisms and specific pathways by which TRPVs act to alleviate nerve injuries.

\section{Contents}

1. Introduction

2. Methods

3. Role of TRPV1 in cerebral ischemic injury

4. Role of TRPV2 in cerebral ischemic injury

5. Role of TRPV4 in cerebral ischemic injury

6. Discussion

7. Conclusions

\section{Introduction}

Ischemic stroke is a common clinical disease caused by an insufficient blood supply to the brain. Characterized by high morbidity, lethality and mortality, it is the third leading cause of death in the world and the primary cause of death in China $(1,2)$. As such, it is a heavy burden on families and society. The pathobiology of ischemic stroke is complicated and involves energy metabolism disorders, peroxidation, calcium ion $\left(\mathrm{Ca}^{2+}\right)$ overload, neurotoxicity, as well as other complications (3). Ion homeostasis and osmotic pressure are abnormal in the early stage of cerebral ischemia and destroy the dynamic balance of the blood-brain barrier (BBB), which causes an overflow of plasma components into the brain. Subsequently, endogenous edema of the blood vessels and a series of cascade reactions occur, which aggravate the neurons and any existing brain injury (4). Following cerebral ischemia, ATP synthesis disorders further result in insufficient energy supply, metabolism and homeostasis $(5,6)$. Additionally, blood flow to the brain is markedly reduced after a few minutes of cerebral ischemia, which causes irreversible brain damage to varying degrees, including cell necrosis and even neurovascular unit injury $(7,8)$.

Transient receptor potential (TRP) channels consist of a group of 28-30 TRP proteins, which are closely related structurally and form ion channels in the membranes of numerous animals (9). A number of studies in previous years have revealed that the expression and activity of various TRP 
channel proteins are altered during the course of cerebral ischemia-reperfusion (IR) injury $(10,11)$ and a large number of in vivo and in vitro experiments have demonstrated that multiple pathways interfering with the regulation of the TRP channel effectively prevent IR injury $(12,13)$. Studies have demonstrated that the pathogenesis of cerebral ischemia is closely associated with $\mathrm{Ca}^{2+}(14)$ and that members of the TRP vanilloid (TRPV) family have high permeability to $\mathrm{Ca}^{2+}(15)$. Moreover, cerebral ischemic injury has been associated with TRPVs, which indicates that the regulation of TRPVs could affect the repair of damaged neurons (16).

TRPVs are divided into TRPV1, TRPV2, TRPV3, TRPV4, TRPV5 and TRPV6. Table I displays their functions and organization. TRPV1 to TRPV4 belong to the non-selective ion channel group, with TRPV1, TRPV2 and TRPV4 distributed predominantly in the central nervous system (CNS) and TRPV3 being distributed in the skin. TRPV5 and TRPV6 are selective osmotic $\mathrm{Ca}^{2+}$ channels, and TRPV5 is important in $\mathrm{Ca}^{2+}$ reabsorption due to its expression in renal epithelial cells (17). TRPV6 is predominantly expressed in the intestinal tract of animals and exerts an effect on intestinal $\mathrm{Ca}^{2+}$ absorption (18). Evidence indicates that there is a mutual relationship between ischemic injury and TRPVs (10-13). Due to the distribution of TRPV3, TRPV5 and TRPV6, this paper focuses on the function of TRPV1, TRPV2 and TRPV4, which are highly expressed in the brain, and their role in cerebral ischemia or under hypoxic conditions. Therefore, the aim of the present review is to provide new strategies for research and to advance the clinical treatment of strokes by reviewing the existing literature on TRPVs and cerebral ischemia.

\section{Methods}

PubMed (https://pubmed.ncbi.nlm.nih.gov/) and China National Knowledge Infrastructure databases (http://www. cnkiTabet) were searched using the following search terms: TRPV OR transient receptor potential vanilloid OR TRPV1 OR TRPV2 OR TRPV4 AND cerebral ischemia OR cerebral ischemia-reperfusion injury from the time of construction of the library to January 2020. Based on the title, abstract and other information in the literature, such as models and the relationship to ischemic injury, certain search results were excluded. The included literature relating to TRPV1, TRPV2 or TRPV4 were read carefully and individually. Finally, the roles of TRPV1, TRPV2 and TRPV4 in cerebral ischemia were reviewed and summarized.

\section{Role of TRPV1 in cerebral ischemic injury}

TRPV1 is highly expressed in the CNS and is activated by high temperatures $\left(>43^{\circ} \mathrm{C}\right)$, acidic conditions $(\mathrm{pH}<6.0)$, exogenous stimuli compounds (capsaicin and allyl isothiocyanate) (19) and endogenous stimuli (cannabinoid anandamide, $\mathrm{N}$-oleoyldopamine and $\mathrm{N}$-arachidonic acid-dopamine) $(20,21)$. Neuronal damage is the main cause of brain dysfunction, and a previous study has revealed that activation of TRPV1 confers neuroprotection by enhancing axonal signaling in neurons (22). Autophagy, which promotes neuronal survival and induces neuronal apoptosis or cell death, is activated during cerebral ischemia. This process occurs several hours after stroke and is key in determining the fate of neurons (23). Apoptosis also requires activation of apoptotic genes, involvement of the mitochondria, release of cytochrome $c$ and activation of caspase family cascades (24). The opposing roles of autophagy may be related to the degree of autophagy activation occurring at different stages of a stroke. Furthermore, moderate autophagy removes damaged organelles and inhibits neuronal apoptosis to exert a protective effect (25). Therefore, inhibition of the development of cell apoptosis appears to be an effective way of reducing the degree of cerebral ischemia or cerebral IR injury (26). Notably, a previous study revealed that, following activation of TRPV1, nerve cell apoptosis under hypoxia/re-oxygenation was clearly improved following an influx of $\mathrm{Ca}^{2+}(27)$.

TRPV1 receptors are present in cerebrovascular endothelial cells and are activated by capsaicin, resulting in an influx of $\mathrm{Ca}^{2+}(28)$. Notably, the TRPV1 channel, which is activated by capsaicin, reduces the infarct volume in IR rats, improving their motor coordination and neurobehavioral scores (29). Evodiamine induces protective autophagy via the $\mathrm{Ca}^{2+} / \mathrm{c}-\mathrm{JNK}$ pathway (30); however, capsicum also reduces $\mathrm{Ca}^{2+}$ influx and increases apoptosis (31). In addition, autophagy is activated by various stress factors associated with mediated cytotoxic damage processes (32). Notably, contrary evidence also exists demonstrating that a TRPV1 antagonist (capsazepine) reduces infarct size without influencing cerebral blood flow in a middle cerebral artery occlusion (MCAO) model (33). Wild-type and TRPV1-knockout mice with cerebral ischemia exhibited no differences in the increased number of ionized calcium binding adaptor molecule 1-positive microglia/macrophages, glial fibrillary acidic protein-positive astrocytes or granulocyte receptor-1-positive neutrophils (33). Together, these outcomes suggest that TRPV1 activated by ischemic injury could cause neurological and motor deficits, as well as brain infarction, but the exact cause remains unclear. Therefore, the relationship between TRPV1 and injuries has not yet been definitively determined. Based on the existing literature, it is apparent that the varying outcomes cannot prove that TRPV1 has biphasic directional regulation. Studies to date have demonstrated that regulation of the TRPV1 channel alters neurological function scores and relieves ischemic injury by regulating cerebral blood flow, inhibiting nerve excitation, promoting anti-inflammation and inducing hypothermia (34-55).

TRPVI in blood vessels. A previous study established that TRPV1 mediates endothelium-dependent and -independent vasodilation, and participates in the regulation of cerebral blood flow (34), thereby reducing ischemia and hypoxia neuronal injury. A study revealed that cannabinoids could activate the TRPV1 receptor at the neuron ends, which resulted in the release of calcitonin gene-related peptide (CGRP), the hyperpolarization of smooth muscle cells to activate $\mathrm{K}^{+}$channels and vasodilation (35). A similar study has also demonstrated that activation of TRPV1 in perivascular sensory nerve endings promotes the release of CGRP and vasodilation; however, the vasodilation was significantly reduced in knockout TRPV1 mice (36). Further studies revealed that activation of TRPV1 induced extracellular $\mathrm{Ca}^{2+}$ influxes with nitric oxide (NO) production or vasodilation (37-39). 
Table I. Functions and distributions of TRPV1 to TRPV6.

A, Non-selective ion channels

\begin{tabular}{lll}
\hline Channel & \multicolumn{1}{c}{ Function } & \multicolumn{1}{c}{ Distribution } \\
\hline TRPV1 & Vanilloid receptor and noxious thermosensor $\left(43^{\circ} \mathrm{C}\right)$ & $\mathrm{CNS}$, PNS \\
TRPV2 & Osmosis and noxious heat thermosensor $\left(52^{\circ} \mathrm{C}\right)$ & $\mathrm{CNS}$, spleen, lung \\
TRPV3 & Warmth sensor channel $\left(33-39^{\circ} \mathrm{C}\right)$ & Skin, CNS, PNS \\
TRPV4 & Osmosis and warmth sensor channel $\left(27-34^{\circ} \mathrm{C}\right)$ & $\mathrm{CNS}$, internal organs \\
\hline
\end{tabular}

$\mathrm{B}$, Selective osmotic $\mathrm{Ca}^{2+}$ channels

\begin{tabular}{lll}
\hline Channel & Function & Distribution \\
\hline TRPV5 & Calcium-selective channel & Intestine, kidney, placenta \\
TRPV6 & Calcium-selective channel & Kidney, intestine \\
\hline
\end{tabular}

TRPV, transient receptor potential vanilloid; CNS, central nervous system; PNS, peripheral nervous system. More information can be obtained from this reference (9).

TRPV1 in neurotoxicity. Following cerebral ischemia, the release of glutamates into the presynaptic membranes is increased. Glutamate aggregation in the synaptic cleft and activation of glutamate receptors results in an excessive $\mathrm{Ca}^{2+}$ influx, which causes cell death or excitotoxicity (40). Previous in vitro studies have revealed that capsaicin reduces glutamate-induced $\mathrm{Ca}^{2+}$ influx resulting from cortical neuron excitotoxicity and levels of phospho-NMDA receptor 1 (GluN) 1, GluN2B and the N-methyl-D-aspartate receptor (NMDAR). Furthermore, capsaicin can reduce infarct volume, and improve neurobehavioral scores and motor coordination in IR rats $(29,38)$. These studies suggested that capsaicin exerted a neuroprotective effect in cortical neurons via TRPV1.

TRPVI in inflammation. Inflammation is important in the process of cerebral ischemic injury. Following cerebral ischemia, leukocyte aggregation and microglia activation leads to the production of a variety of pro-inflammatory cytokines. Moreover, microglia contribute to inflammation of the brain, particularly in the ischemic penumbra. In addition, endothelial cells, astrocytes and neurons secrete pro-inflammatory cytokines following ischemic injury. The combination of these inflammatory cells and pro-inflammatory cytokines results in further damage to the neurons (41). Preventing the inflammatory response is therefore another important approach that is essential for protecting the brain. A previous study demonstrated that TRPV1 inhibition decreased levels of TNF- $\alpha$ and IL-10 in plasma, which reduced infarction size, thus conferring a neuroprotective effect (42). Another study revealed that inhibiting TRPV1 exerted neuroprotection in rats with cerebral IR injury, which was partially associated with TRPV1-mediated antioxidant stress and anti-inflammation due to inhibiting p38 MAPK activation (43). TRPV1 promotes activation of astrocytes and the release of astrocyte-derived IL-1 $\beta$, predominantly via the Janus kinase 2 -signal transducer and activator of transcription 3 signaling pathway, and activation of the nucleotide-binding oligomerization domain-like receptor protein 3 inflammasome in hypoxic-ischemic encephalopathy (44). Overall, these comprehensive analyses suggest that the expression of TRPV1 is closely associated with the inflammatory response and that regulation of TRPV1 may reduce the inflammation induced by ischemic injury (42).

TRPV1 in hypothermia. A role for TRPV1 in the regulation of body temperature has also previously been described (45). TRPV1 is tonically active in vivo. Numerous TRPV1 antagonists are used as analgesics, but are accompanied by hyperthermia, which indicates that TRPV1 activation could regulate body temperature (45). This explains the propensity of the TRPV1 agonist capsaicin to cause sweating in order to reduce body temperature. Without such signals, the body overheats. In a previous study, it was found that tonically active TRPV1 channels are presented in the viscera and confer a suppressive effect on body temperature (46). Body temperature maintenance was also recently proposed to be the predominant function of TRPV1 (47) and additional studies have determined that hypothermia inhibits neuronal cell apoptosis during ischemic injury (48-50). The activation of TRPV1 triggers an autonomic nerve reaction that promotes heat loss in the hypothalamus; body temperature and oxygen metabolism are reduced, and blood oxygen saturation is increased, which alleviates nerve injury (51-53). Additionally, the activation of TRPV1 blocks or alleviates cell excitotoxicity and reduces inflammation factors and free radical levels. Studies have determined that TRPV1 agonists induce hypothermia, thus reducing infarct size and improving neurological function scores in IR animals $(54,55)$. These observations suggest that activation or inhibition of TRPV1 promotes a neuroprotective effect via both positive and negative feedback regulation.

\section{Role of TRPV2 in cerebral ischemic injury}

Structurally, TRPV2 is $50 \%$ homologous to TRPV1; however, the activation temperature for TRPV2 channel thermal 
stimulation is higher $\left(\geq 52^{\circ} \mathrm{C}\right)$ compared with TRPV1 (56). In addition, TRPV2 is activated by various mechanical and chemical stimuli, such as osmotic pressure. Studies have revealed that TRPV2, which is associated with $\mathrm{Ca}^{2+}$ transport, is abundantly expressed in the cell membranes of astrocytes $(57,58)$. TRPV2 is also present in neurons and other non-neuronal tissues, such as the heart and lungs, and it serves an important role in basic cellular functions, such as cell contraction, proliferation and death (59). The main expression sites, however, are neurons. For neurons, neurite outgrowth is the key to the formation of functional circuits during neuronal development (60). A previous study demonstrated that TRPV2 increases the expression levels of nerve growth factor (NGF) and upregulates $\mathrm{Ca}^{2+}$ permeability via an MAPK pathway, thereby activating the extracellular regulated kinase (ERK) signaling pathway to enhance the outgrowth of neurites (61). $\mathrm{Ca}^{2+}$ is also increased in astrocytes following oxygen-glucose deprivation and re-oxygenation (OGD/R) treatment (62). Moreover, blocking TRPV2 increases the synthesis and secretion of NGF, and promotes astrocyte proliferation via the MAPK-JNK signaling pathway. Notably, activation of TRPV2 induces the release of NGF, and protects neurons and blood vessels via a JNK-dependent pathway (63). Furthermore, a TRPV2 agonist induced proliferation, migration and tubulogenesis, as well as increased transendothelial electrical resistance in human brain endothelial cells, which could regulate the function of the BBB (64). These contradictory experimental results support the conclusion that TRPV2 serves an important role in promoting NGF synthesis during ischemic stroke.

\section{Role of TRPV4 in cerebral ischemic injury}

TRPV4 is another non-selective $\mathrm{Ca}^{2+}$ channel that is expressed in various tissues, such as the CNS, heart, liver, kidney and lungs. In the CNS, TRPV4 is activated by multiple stimuli and is distributed among neurons, glial cells, cerebral vascular smooth muscle and the endothelial cells of the brain (65). Studies have demonstrated that TRPV4 serves an important regulatory role in a variety of physiological and pathological processes $(66,67)$. Additionally, TRPV4 is activated during cerebral ischemia when microcirculatory disorders and energy deficiency leads to a change in cytotoxic edema and cell membrane tension $(68,69)$. This suggests that TRPV4 might mediate cerebral ischemic injury. In addition, inhibition of TRPV4 has been shown to reduce both the expression of MMP-9 and the permeability of the BBB in IR rats (70). A TRPV4 inhibitor also exerted a protective effect on hippocampal carbonic anhydrase 1 (CA1) neuronal injury caused by OGD (71). Similarly, excitatory amino acids are increased in the extracellular space following cerebral ischemia, where oxygen free radicals and factors are also found $(72,73)$. In addition, abnormal expression of genes controlling apoptosis can lead to infarction and apoptosis (74). Furthermore, TRPV4-mediated $\mathrm{Ca}^{2+}$ influx promotes apoptosis and necrosis in OGD/R models (74). Previous studies demonstrated that infarction size increases following cerebral ischemia, and TRPV4 and phosphorylated (p)-ERK levels increase while p-Akt is downregulated, which can be blocked by TRPV4 inhibitors $(75,76)$. Studies have also found that regulating $\mathrm{Ca}^{2+}$, preventing inflammation and inhibiting neurotoxicity are the predominant mechanisms by which TRPV4 protects neurons $(77,78)$. Overall, the mechanisms of TRPV4 and TRPV1 in cerebral ischemia are highly similar.

TRPV4 in $\mathrm{Ca}^{2+}$ influx. $\mathrm{Ca}^{2+}$ serves an important role in cerebral ischemia. A large quantity of $\mathrm{Ca}^{2+}$ enters the cells via the NMDAR, which causes $\mathrm{Ca}^{2+}$ overload and cell damage (79). TRPV4 is clearly involved in the pathological process of cerebral IR injury and, when overexpressed, causes $\mathrm{Ca}^{2+}$ influx following ischemic injury, thus increasing the release of presynaptic neurotransmitters (13). TRPV4 also improves NMDAR function to reduce neurotoxicity by increasing glutamates and overloading $\mathrm{Ca}^{2+}$ to relieve ischemic injury. Following hypoxia-ischemia, TRPV4 levels are significantly increased in astrocytes of the hippocampal CA1, thus promoting the proliferation of reactive astrocytes (13). Furthermore, TRPV4 agonists cause intracellular $\mathrm{Ca}^{2+}$ and cation currents, which are blocked by extracellular $\mathrm{Ca}^{2+}$ scavengers or TRPV4 antagonists (13). This suggests that TRPV4 is involved in $\mathrm{Ca}^{2+}$ influx in ischemic-reactive astrocytes. Preservation of microcirculation and BBB function shortly after reperfusion is the key neuroprotective role of TRPV4 inhibition, suggesting that TRPV4 contributed to post-ischemic brain injury (80).

TRPV4 in inflammation and apoptosis. TRPV4 is a non-selective, calcium-permeable cation channel that serves a critical role in cerebral perfusion and inflammation (81). It has been reported that TRPV4 is activated in choroid plexus epithelia and cerebral ischemia by cytokines and inflammatory mediators, such as TNF- $\alpha$, IL-1 $\beta$ and TGF- $\beta 1(82,83)$. A previous study demonstrated that the expression of TRPV4 is significantly upregulated in MCAO rats, and that a TRPV4 inhibitor reduces TNF- $\alpha$ and IL- $1 \beta$ levels to alleviate astrocyte OGD injury (84). Activation of TRPV4 induces apoptosis by downregulating the PI3K/Akt signaling pathway and upregulating the p38 MAPK signaling pathway, which are involved in cerebral ischemic injury (75). The TRPV4 antagonist reduced brain infarction following reperfusion for $48 \mathrm{~h}$ in MCAO mice, which attenuated a decrease in the p-Akt and $\mathrm{Bcl}-2 / \mathrm{Bax}$ protein ratio and an increase in p-p38 MAPK and cleaved caspase-3 protein levels (75).

TRPV4 in excitotoxicity. Following cerebral ischemia, the damaged $\mathrm{BBB}$ and extracellular $\mathrm{Ca}^{2+}$ influx result in neurotoxicity. TRPV4 is similar to TRPV1 in regard to their role in neurotoxicity. Previous studies have demonstrated that TRPV4 inhibits neurotoxicity via the NMDAR 2B $(13,79)$. Another study indicated that TRPV4 inhibitors block the neurotransmitter $\gamma$-aminobutyric acid of hippocampal CA1 pyramidal neurons via the adenosine 5'-monophosphate activated protein kinase (AMPK)-PI3K-Akt pathways to inhibit neuronal hyperexcitability (85). Together, the existing research indicates that blocking TRPV4 may regulate $\mathrm{Ca}^{2+}$ influxes, thus inhibiting the inflammatory response, autophagy and apoptosis following cerebral ischemia.

\section{Discussion}

The mechanisms involved in brain protection conferred by TRPV1, TRPV2 and TRPV4 are presented in Table II. 
Table II. Mechanisms involved in reducing ischemic injury via TRPV1, TRPV2 and TRPV4.

\begin{tabular}{|c|c|c|c|c|c|}
\hline Mechanism & $\begin{array}{c}\text { TRPV1 } \\
\text { (channel activation) }\end{array}$ & $\begin{array}{c}\text { TRPV1 } \\
\text { (channel inhibition) }\end{array}$ & TRPV1-KO & $\begin{array}{c}\text { TRPV2 } \\
\text { (channel inhibition) }\end{array}$ & $\begin{array}{c}\text { TRPV4 } \\
\text { (channel inhibition) }\end{array}$ \\
\hline $\mathrm{Ca}^{2+}$ influx & - & Inhibition $(29,38,40)$ & - & Inhibition $(61,62)$ & Inhibition (79-80) \\
\hline Inflammatory reaction & - & Inhibition (41-44) & NR & NR & Inhibition (81-84) \\
\hline Neurotoxicity & - & Inhibition $(29,38)$ & - & NR & Inhibition $(13,79,85)$ \\
\hline Neuron growth & NR & NR & NR & Activation $(61,63)$ & NR \\
\hline $\begin{array}{l}\text { Hypothermic brain } \\
\text { protection }\end{array}$ & Activation (45-55) & - & - & NR & NR \\
\hline Vasodilation & - & Activation (34-39) & - & NR & NR \\
\hline
\end{tabular}

TRPV, transient receptor potential vanilloid; -, no effect; NR, no reported studies in the current review.

Numerous studies have reported that the activation of TRPV1 attenuates excitotoxicity injury to inhibit $\mathrm{Ca}^{2+}$ influxes, and glutamate-induced neuronal excitability and neuronal death $(29,38,40)$. Additionally, activation of TRPV1 increases endothelial nitric oxide synthase (eNOS) phosphorylation to improve vasodilation and induce hypothermic brain protection, ultimately reducing cerebral infarction size and neurological scores (37-39). While capsaicin-induced effects can be reduced or reversed in TRPV1-knockout mice, inhibition of TRPV1 has also been revealed to inhibit the release of inflammatory factors, thus inducing hypothermic brain protection, reducing cerebral infarction size and improving neuron behavior (41-46). This contradiction of whether TRPV1 was activated or inhibited to protect brain injuries may be due to the extreme complexity of the pathogenesis of cerebral ischemia and the lack of details regarding the role of TRPV1 in the mechanism of cerebral ischemia.

These contrasting outcomes may be the result of the following: i) Variable TRPV1 agonists and inhibitors. In the collected literature, TRPV1 agonists included capsaicin (29), evodiamine (30) and dihydrocapsaicin (DHC) $(51,54)$. The TRPV1 inhibitors included capsazepine (33) and AMG-9810 (42). Those drugs may have slightly different effects via different pathways. ii) Variable dosages may impact the results. For example, the dosages of capsaicin used were 1 or $3 \mathrm{nmol}$ (29) or $1 \mathrm{mg} / \mathrm{kg}$ (42), and Huang et al (29) found that 1 or 3 nmol capsaicin reduced cerebral infarction size and improved motor function. Hakimizadeh et al (42) used capsaicin in measures of $0.1,0.5$ or $1 \mathrm{mg} / \mathrm{kg}$, and Cao et al (51) used $1.25 \mathrm{mg} / \mathrm{kg}$ DHC with no obvious neuroprotective effect. Furthermore, the study by Cao et al indicated that a TRPV1 agonist $(25 \mathrm{mg} / \mathrm{kg}$ rinvanil) induced hypothermia with a neuroprotective effect on ischemic brain injury. Meanwhile, a high dosage of rinvanil $(50 \mathrm{mg} / \mathrm{kg})$ demonstrated no significant effect on brain injury $(33,54)$. The dosage of capsazepine (TRPV1 inhibitor) was $20 \mathrm{nmol}$ (33) and that of AMG-9810 was $0.5 \mathrm{mg} / \mathrm{kg}$ (42). In addition, the varied selection of animal models, including Sprague-Dawley rats, Wistar rats and C57B/L6 mice among others, may have impacted the results. Therefore, due to the differing results, a more rigorous experimental design is required, along with an increased number of samples, to clarify the mechanisms by which TRPV1 channels confer their effects in order to provide a solid theoretical foundation for the development of therapeutic strategies targeting cerebral ischemia.

The existing literature has established that TRPV1 and TRPV4 demonstrate similar effects on $\mathrm{Ca}^{2+}$ influx, inflammation and neurotoxicity. The mode of action for TRPV2 in regard to relieving ischemic injury is unique, as it includes four main signaling pathways, $\mathrm{Ca}^{2+} / \mathrm{JNK}$, PI3K/Akt, MAPK and $\mathrm{Ca}^{2+}$. Table III compares the results of each pathway. TRPV1 and TRPV4 serve similar roles in blocking $\mathrm{Ca}^{2+}$ influx, inhibiting the inflammatory response and reducing neurotoxicity. An ion channel blocker (such as capsazepine) effectively reduces $\mathrm{Ca}^{2+}$ influx, the inflammatory response and neurotoxicity induced by cerebral ischemia injury, the mechanism of which is related to the MAPK-JNK pathways. In contrast to the pharmacological effects of TRPV4, TRPV1 is also likely a sensor that participates in the regulation of body temperature, which could serve to decrease IR injury, but the pathway by which it functions is unique. When TRPV1 is activated, body temperature and metabolism are reduced. The existing literature has established that TRPV1 activation reduces IR injury $(54,55)$; however, there have been reports indicating conflicting results, as inhibition of TRPV1 resulted in a decrease in body temperature, thus reducing IR injury (42). As such, it is clear that TRPV1 regulates body temperature and this is a fundamental difference in relation to TRPV4.

TRPVs are important ion channels associated with ischemic stroke. A comprehensive schematic detailing the correlation among the three TRPVs in relation to ischemia or hypoxic injury is provided in Fig. 1, which includes three main signaling pathways, p38 MAPK, JNK and PI3K/Akt. The MAPK pathway mediates the proliferation, differentiation and apoptosis of neuronal cells under different pathophysiological conditions and is related to neurological diseases $(86,87)$. It has been revealed that the MAPK pathway is a key pathway involved in the protection of the BBB (88-90). Furthermore, JNK and p38 are key proteins in the MAPK pathway. It has been confirmed that the JNK/p38 MAPK pathway involves almost all physiological and pathological processes of ischemic and IR injury, and it is one of the most critical pathways involved in BBB injuries (89). The activity of p38 in neurons is significantly enhanced in the ischemic region of rats exhibiting IR injury, and the expression of apoptosis genes, such as Bcl and Bax, is increased, thereby inducing 
Table III. Relief of ischemic injury by TRPV1, TRPV2 and TRPV4.

Channel Process of relieving ischemic injury

TRPV1 Therapeutic hypothermia, $\mathrm{Ca}^{2+}$, NMDAR, JNK pathway

TRPV2 MAPK-JNK signaling pathway, JNK-dependent pathway

TRPV4 $\quad \mathrm{Ca}^{2+}$, NMDAR, Akt signaling pathway, PI3K/Akt pathway, p38MAPK pathway, AMPK-PKC pathway

TRPV, transient receptor potential vanilloid; NMDAR, N-methyl-D-aspartic acid receptor; AMPK, adenosine 5'-monophosphate activated protein kinase.

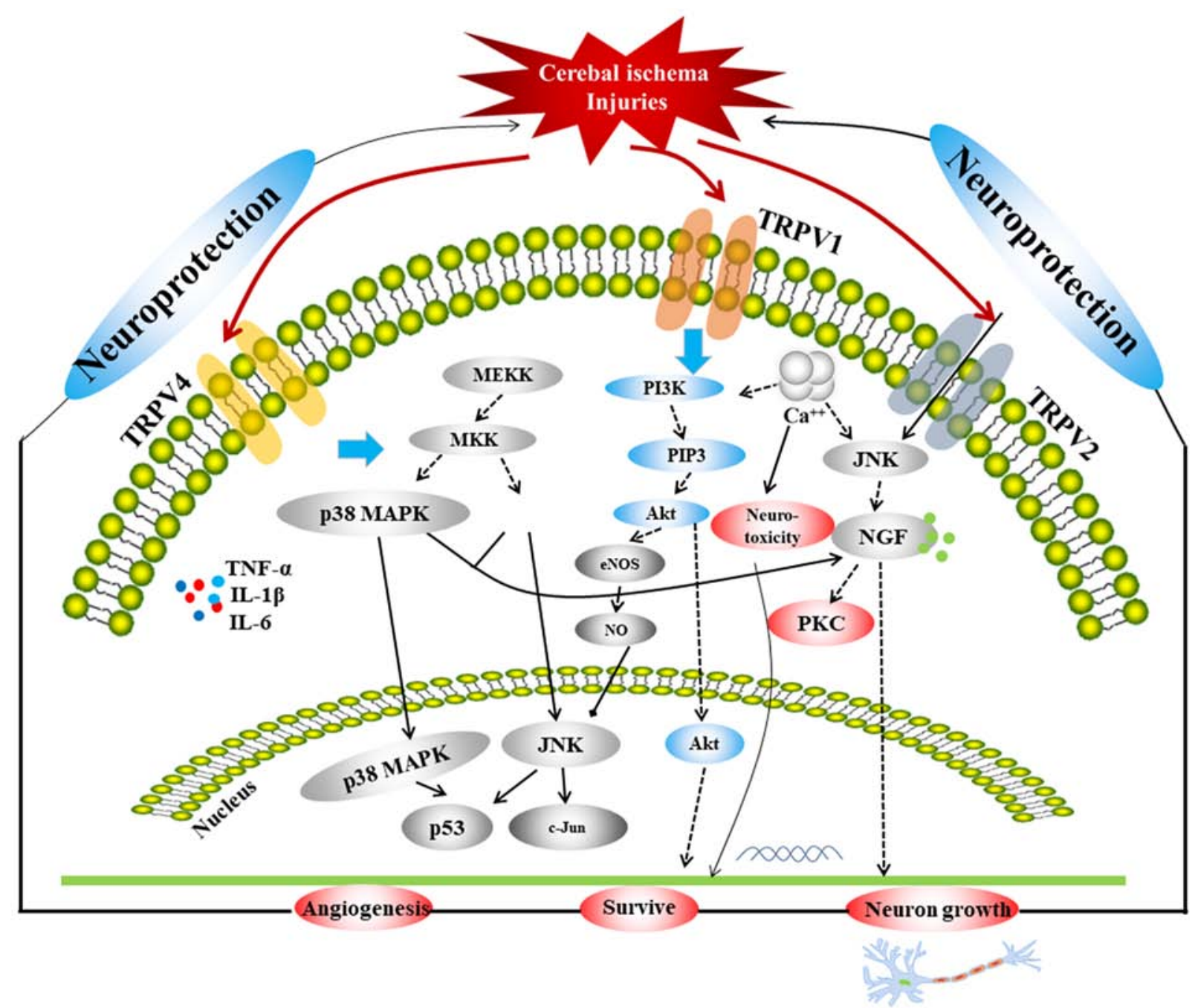

Figure 1. Associations among the TRPV1, TRPV2 and TRPV4 channels in regard to cerebral ischemia injuries. TRPV, transient receptor potential vanilloid; MEKK, MAP kinase kinase kinase; MKK, mitogen-activated protein kinase kinase; PIP3, phosphatidylinositol (3-5)-trisphosphate; eNOS, endothelial nitric oxide synthase; NO, nitric oxide; NGF, nerve growth factor.

the apoptosis of neuronal cells (91). A previous study determined that under a variety of stimuli, the p38 MAPK pathway is involved in mediating the production of inducible NO synthase (92). Following cerebral IR, a large quantity of NO is produced, which regulates MMP-9 through guanylate cyclase (93), promoting the development of ischemic brain injuries. In addition, a previous study demonstrated that the JNK pathway, which is a MAPK signaling pathway, induces apoptosis following IR injury via the mitochondrial and death receptor pathways (94); as such, inhibiting the JNK pathway could protect neurons from injury. The PI3K/Akt signaling pathway is widely distributed in various cells and is a signal transduction pathway involved in cell survival, proliferation and differentiation regulation (95-97). Activation of the PI3K/Akt signaling pathway inhibits apoptosis via multiple pathways, reduces IR damage and exerts neuroprotective effects $(98,99)$. A previous study found that in the development of ischemic brain injury, the PI3K/Akt cell survival signaling 
pathway predominates during the early stage, whereas the JNK apoptotic signaling pathway predominates during the later stage in MCAO rats. The expression of p-Akt (Ser473) in the penumbra was significantly downregulated $0.5 \mathrm{~h}$ after ischemia, significantly upregulated at $1.5-5 \mathrm{~h}$, downregulated at 9-24 $\mathrm{h}$ and returned to baseline at $48 \mathrm{~h}$, after which the JNK apoptosis signaling pathway began to dominate (100). Furthermore, p-Akt expression in neurons is increased $1-3 \mathrm{~h}$ after IR injury, which indicates that the PI3K/Akt signaling pathway is involved in the early-stage stress response (101). These three pathways are linked by the TRPVs, and are closely associated with autophagy and apoptosis; therefore, they serve an important regulatory role in cerebral ischemic injury.

$\mathrm{Ca}^{2+}$ also serves a pivotal role in cerebral ischemia, as intracellular $\mathrm{Ca}^{2+}$ overloaded via NMDAR triggers a series of harmful events and is the final common pathway leading to nerve cell death (102). Therefore, inhibition of $\mathrm{Ca}^{2+}$ overload could also be an important approach for reducing the extent of cerebral ischemic injury. A series of cascades occur due to increased $\mathrm{Ca}^{2+}$ influx and glutamate levels following cerebral ischemia, which are important factors that serve to aggravate ischemic or IR injury. Following cerebral ischemia, increased levels of glutamate activate the glutamate receptors and $\mathrm{Ca}^{2+}$ influx occurs, which could induce neurotoxicity via NMDAR. TRPV1 and TRPV4 inhibit $\mathrm{Ca}^{2+}$ influx and reduce neurotoxicity, which might be related to the inhibition of NMDAR expression and the regulation of the AMPK pathway in the relief of ischemic injury.

TRPV1 and TRPV4 inhibit inflammatory responses by reducing inflammatory factors and by linking the JNK, Akt and ERK pathways to promote apoptosis and autophagy $(43,75,76)$. Therapeutic hypothermia induced by TRPV1 reduces the consumption of sugar and oxygen, and the metabolism of brain cells to exert additional neuroprotective effects (45-57). TRPV1 also enhances vasodilation to alleviate ischemic injury via the AMPK-eNOS pathway (37-39). The mechanism of TRPV2 in attenuating ischemic injury is different from that of TRPV1 or TRPV4 in that it promotes the release of NGF, thereby promoting neuronal growth via the MAPK-JNK/ERK pathways $(61,63)$.

In the present review, the reason for the different effects of TRPV1 and TRPV2, (which are non-selective ion channels) were analyzed. Previous studies demonstrated that $\mathrm{Ca}^{2+}$ serves an important role in ischemia and IR injuries $(28,31)$. Therefore, the majority of studies conducted related experiments, which revealed that TRPV1 and TRPV2 are highly permeable to $\mathrm{Ca}^{2+}(29,38,61)$. However, their roles in injuries are vastly different, which the present study hypothesizes is due to their different expression sites. TRPV1 is expressed in numerous cell types, such as neural, vascular endothelial and glial cells, whereas TRPV2 is primarily expressed in neural cells. Thus, studies on TRPV1, including blood vessels, neurotoxicity and inflammation are extensive. There were few studies on TRPV2 and ischemia or ischemic reperfusion. The existing data predominantly refer to NGF and TRPV2. NGF is a key factor in neuron growth, which might be the entry point for studying them. In future studies, further investigation into other factors or pathways and how they are associated with TRPV2 could be investigated, which would enhance research data and provide a basis for the treatment of cerebral ischemia. Among the TRPVs, TRPV1 is expressed in cerebrovascular endothelial cells, neurons and astrocytes (103), and the TRPV1 channel has been revealed to be a potential target influencing the efficacy of treating strokes (16).

\section{Conclusions}

In addition to their crucial function in regulating $\mathrm{Ca}^{2+}$ influx, TRPVs also exert a neuroprotective effect via other signaling pathways, such as the p38 MAPK, JNK and PI3K-Akt signaling pathways. The physiological effects accompanying the TRPV channels and their associated signal transduction pathways still require further investigation. Furthermore, additional in vivo and in vitro experiments are required to rigorously evaluate the role of TRPVs in neurological injury following cerebral ischemia, as well as to provide targets for the development of novel drugs and to inform strategies for the clinical treatment of cerebral ischemia, as the pathogenesis of cerebral ischemic injury is complex. In the future, the related pathways of angiogenesis and TRPVs require further investigation to determine whether the mechanism regulating TRPVs can help increase axon signal transduction in neurons and enhance brain protection.

\section{Acknowledgements}

Not applicable.

\section{Funding}

The present study was supported by the National Natural Science Foundation of China (grant nos. 81873023 and 81473371), the Innovation Team in Chengdu University of Traditional Chinese Medicine (grant no. CXTD2018004) and the Open Research Fund of the Key Laboratory of Southwestern Characteristic Chinese Medicine Resources, Chengdu University of Traditional Chinese Medicine (grant no. 2020XSGG025).

\section{Availability of data and materials}

Not applicable.

\section{Authors' contributions}

QX and RM collected the data, wrote the manuscript and confirm the authenticity of all the raw data. JW and $\mathrm{HC}$ collected literature and oversaw article writing. XG and HL accessed the literature and edited the manuscript. All authors read and approved the final version of the manuscript.

\section{Ethics approval and consent to participate}

Not applicable.

\section{Patient consent for publication}

Not applicable.

\section{Competing interests}

The authors declare that they have no competing interests. 


\section{References}

1. Vos T, Allen C, Arora M, Barber RM, Bhutta Z, Brown A, Carter AR, Charlson FJ, Chen A, Coggeshall M, et al: Global, regional, and national incidence, prevalence, and years lived with disability for 310 diseases and injuries, 1990-2015: A systematic analysis for the global burden of disease study 2015. Lancet 388: 1545-1602, 2016.

2. Zhou M, Wang H, Zhu J, Chen W, Wang L, Liu S, Li Y, Wang L, Liu Y, Yin P, et al: Cause-specific mortality for 240 causes in China during 1990-2013: A systematic subnational analysis for the global burden of disease study 2013. Lancet 387: 251-272, $10015,2016$.

3. Huang ZY, ZhangXX, Sun WL, Chen C, Li DF, Fang J, Fu MH, Liu QS, Yan TH and Li SJ: Research progress of inflammation reaction related to endoplasmic reticulum stress in ischemic endoplasmic reticulum stress. Chin Pharmacol Bull 31: 23-26, 2015 (In Chinese).

4. Dirnagl U, Iadecola C and Moskowitz MA: Pathobiology of ischaemic stroke: An integrated view. Trends Neurosci 22: 391-397, 1999.

5. Kovac S, Kostova Dinkova AT, Herrmann AM, Melzer N, Meuth SG and Gorji A: Metabolic and homeostatic changes in seizures and acquired epilepsy-mitochondria, calcium dynamics and reactive oxygen species. Int J Mol Sci 18: 1935, 2017.

6. Fahrner JA, Liu R, Perry MS, Klein J and Chan DC: A novel de novo dominant negative mutation in DNM1L impairs mitochondrial fission and presents as childhood epileptic encephalopathy. Am J Med Genet A 170: 2002-2011, 2016.

7. Chan PH: Reactive oxygen radicals in signaling and damage in the ischemic brain. J Cereb Blood Flow Metab 21: 2-14, 2001.

8. Chan PH: Role of oxidants in ischemic brain damage. Stroke 27: 1124-1129, 1996.

9. Venkatachalam K and Montell C: TRP channels. Annu Rev Biochem 76: 387-417, 2007.

10. Rakers C, Schmid M and Petzold GC: TRPV4 channels contribute to calcium transients in astrocytes and neurons during peri-infarct depolarizations in a stroke model. Glia 65 : 1550-1561, 2017.

11. Han J, Xu HH, Chen XL, Hu HR, Hu KM, Chen ZW and He GW: Total flavone of rhododendron improves cerebral ischemia injury by activating vascular TRPV4 to induce endothelium-derived hyperpolarizing factor-mediated responses. Evid Based Complement Alternat Med 2018: 8919867, 2018.

12. Seki T, Goto K, Kiyohara K, Kansui Y, Murakami N, Haga Y, Ohtsubo T, Matsumura K and Kitazono T: Downregulation of endothelial transient receptor potential vanilloid type 4 channel and small-conductance of $\mathrm{Ca} 2+$-activated $\mathrm{K}+$ channels underpins impaired endothelium-dependent hyperpolarization in hypertension. Hypertension 69: 143-153, 2017.

13. Li L, Qu W, Zhou L, Lu Z, Jie P, Chen L and Chen L: Activation of transient receptor potential vanilloid 4 increases NMDA-activated current in hippocampal pyramidal neurons. Front Cell Neurosci 7: 17, 2013.

14. Lau A and Tymianski M: Glutamate receptors, neurotoxicity and neurodegeneration. Pflugers Arch 460: 525-542, 2010.

15. Wong CO, Chen K, Lin YQ, Chao Y, Duraine L, Lu Z, Yoon WH, Sullivan JM, Broadhead GT, Sumner CJ, et al: A TRPV channel in Drosophila motor neurons regulates presynaptic resting $\mathrm{Ca} 2+$ levels, synapse growth, and synaptic transmission. Neuron 84 : 764-777, 2014.

16. Satheesh NJ, Uehara Y, Fedotova J, Pohanka M, Büsselberg D and Kruzliak P: TRPV currents and their role in the nociception and neuroplasticity. Neuropeptides 57: 1-8, 2016.

17. Hoenderop JG, Nilius B and Bindels RJ: Molecular mechanism of active $\mathrm{Ca} 2+$ reabsorption in the distal nephron. Annu Rev Physiol 64: 529-549, 2002.

18. Barley NF, Howard A, Callaghan DO, Legon S and Walters JRF: Epithelial calcium transporter expression in human duodenum. Am J Physiol Gastrointest Liver Physiol 2: G285-G290, 2001.

19. Everaerts W, Gees M, Alpizar YA, Farre R, Leten C, Apetrei A, Dewachter I, van Leuven F, Vennekens R, De Ridder D, et al: The capsaicin receptor TRPV1 is a crucial mediator of the noxious effects of mustard oil. Curr Biol 21: 316-321, 2011.

20. Khairatkar-Joshi N and Szallasi A: TRPV1 antagonists: The challenges for therapeutic targeting. Trends Mol Med 15: 14-22, 2009.

21. Cui M, Honore P, Zhong C, Gauvin D, Mikusa J, Hernandez G, Chandran P, Gomtsyan A, Brown B, Bayburt EK, et al: TRPV1 receptors in the CNS play a key role in broad-spectrum analgesia of TRPV1 antagonists. J Neurosci 26: 9385-9393, 2006.
22. Ward NJ, Ho KW, Lambert WS, Weitlauf C and Calkins DJ: Absence of transient receptor potential vanilloid-1 accelerates stress-induced axonopathy in the optic projection. J Neurosci 34: 3161-3170, 2014.

23. Wen YD, Sheng R, Zhang LS, Han R, Zhang X, Zhang XD, Han F, Fukunaga K and Qin ZH: Neuronal injury in rat model of permanent focal cerebral ischemia is associated with activation of autophagic and lysosomal pathways. Autophagy 4: 762-769, 2008.

24. Khan MM, Ishrat T, Ahmad A, Hoda MN, Khan MB, Khuwaja G, Srivastava P, Raza SS, Islam F and Ahmad S: Sesamin attenuates behavioral, biochemical and histological alterations induced by reversible middle cerebral artery occlusion in the rats. Chem Biol Interact 183: 255-263, 2010.

25. Li L, Tan J, Miao Y, Lei P and Zhang Q: ROS and autophagy: Interactions and molecular regulatory mechanisms. Cell Mol Neurobiol 35: 615-621, 2015. Yang Y, Gao K, Hu Z, Li W, Davies H, Ling S, Rudd JA and Fang M: Autophagy upregulation and apoptosis downregulation in DAHP and triptolide treated cerebral ischemia. Mediators Inflamm 2015: 120198, 2015.

26. Charriaut-Marlangue C: Apoptosis: A target for neuroprotection. Therapie 59: 185-190, 2004.

27. Dai Z, Xiao J, Liu S, Cui L, Hu G and Jiang D: Rutaecarpine inhibits hypoxia/reoxygenation-induced apoptosis in rat hippocampal neurons. Neuropharmacology 55: 1307-1312, 2008.

28. Golech SA, McCarron RM, Chen Y, Bembry J, Lenz F, Mechoulam R, Shohami E and Spatz M: Human brain endothelium: Coexpression and function of vanilloid and endocannabinoid receptors. Brain Res Mol Brain Res 132: 87-92, 2004.

29. Huang M, Cheng G, Tan H, Qin R, Zou Y, Wang Y and Zhang Y: Capsaicin protects cortical neurons against ischemia/reperfusion injury via down-regulating NMDA receptors. Exp Neurol 295: 66-76, 2017.

30. Liu A, Wang SH, Hou SY, Lin CJ, Chiu WT, Hsiao SH, Chen TH and Shih CM: Evodiamine induces transient receptor potential vanilloid-1-mediated protective autophagy in U87-MG astrocytes. Evid Based Complement Alternat Med 2013: 354840, 2013.

31. Wang Z, Sun L, Yu H, Zhang Y, Gong W, Jin H, Zhang L and Liang $\mathrm{H}$ : Binding mode pediction of evodiamine within vanilloid receptor TRPV1. Int J Mol Sci 13: 8958-8969, 2012.

32. Hale AN, Ledbetter DJ, Gawriluk TR and Rucker EB III Autophagy: Regulation and role in development. Autophagy 7: 951-972, 2013.

33. Miyanohara J, Shirakawa H, Sanpei K, Nakagawa $\mathrm{T}$ and Kaneko S: A pathophysiological role of TRPV1 in ischemic injury after transient focal cerebral ischemia in mice. Biochem Biophys Res Commun 467: 478-483, 2015.

34. Yang D, Luo Z, Ma S, Wong WT, Ma L, Zhong J, He H, Zhao Z, Cao T, Yan Z, et al: Activation of TRPV1 by dietary capsaicin improves endothelium-dependent vasorelaxation and prevents hypertension. Cell Metab 12: 130-141, 2010.

35. Breyne $\mathbf{J}$ and Vanheel B: Methanandamide hyperpolarizes gastric arteries by stimulation of TRPV1 receptors on perivascular CGRP containing nerves. J Cardiovase Pharmacol 47: 303-309, 2006

36. Xu X, Wang P, Zhao Z, Cao T, He H, Luo Z, Zhong J, Gao F, Zhu Z, Li L, et al: Activation of transient receptor potential vanilloid 1 by dietary capsaicin delays the onset of stroke in stroke-prone spontaneously hypertensive rats. Stroke 42: 3245-3251, 2011.

37. Ching LC, Chen CY, Su KH, Hou HH, Shyue SK, Kou YR and Lee TS: Implication of AMP-activated protein kinase in transient receptor potential vanilloid type 1-mediated activation of endothelial nitric oxide synthase. Mol Med 18: 805-815, 2012.

38. Hurtado-Zavala JI, Ramachandran B, Ahmed S, Halder R, Bolleyer C, Awasthi A, Stahlberg MA, Wagener RJ, Anderson K, Drenan RM, et al: TRPV1 regulates excitatory innervation of OLM neurons in the hippocampus. Nat Commun 8: 15878, 2017.

39. Zhang MJ, Yin YW, Li BH, Liu Y, Liao SQ, Gao CY, Li JC and Zhang LL: The role of TRPV1 in improving VSMC function and attenuating hypertension. Prog Biophys Mol Biol 117: 212-216, 2015.

40. Szydlowska K and Tymianski M: Calcium, ischemia and excitotoxicity. Cell Calcium 47: 122-129, 2010.

41. Chen L, Liu C, Liu L and Cao X: Changes in osmolality modulate voltage-gated sodium channels in trigeminal ganglion neurons. Neurosci Res 64: 199-207, 2009. 
42. Hakimizadeh E, Shamsizadeh A, Roohbakhsh A, Arababadi MK, Hajizadeh MR, Shariati M, Rahmani MR and Allahtavakoli M: Inhibition of transient receptor potential vanilloid-1 confers neuroprotection, reduces tumor necrosis factor-alpha, and increases IL-10 in a rat stroke model. Fund Clin Pharmacol 31: 420-428, 2017.

43. Long M, Wang Z, Zheng D, Chen J, Tao W, Wang L, Yin N and Chen Z: Electroacupuncture pretreatment elicits neuroprotection against cerebral ischemia-reperfusion injury in rats associated with transient receptor potential vanilloid 1-mediated anti-oxidant stress and anti-inflammation. Inflammation 42 : 1777-1787, 2019

44. Yang XL, Wang X, Shao L, Jiang GT, Min JW, Mei XY, He XH, Liu WH, Huang WX, Peng BW: TRPV1 mediates astrocyte activation and interleukin- $1 \beta$ release induced by hypoxic ischemia (HI). J Neuroinflammation 16: 114, 2019.

45. Gavva NR, Bannon AW, Surapaneni S, Hovland DN Jr, Lehto SG, Gore A, Juan T, Deng H, Han B, Klionsky L, et al: The vanilloid receptor TRPV1 is tonically activated in vivo and involved in body temperature regulation. J Neurosci 27 3366-3374, 2007.

46. Steiner AA, Turek VF, Almeida MC, Burmeister JJ, Oliveira DL, Roberts JL, Bannon AW, Norman MH, Louis JC, Treanor JJ, et al: Nonthermal activation of transient receptor potential vanilloid-1 channels in abdominal viscera tonically inhibits autonomic cold-defense effectors. J Neurosci 27 : 7459-7468, 2007.

47. Gavva NR: Body-temperature maintenance as the predominant function of the vanilloid receptor TRPV1. Trends Pharmacol Sci 29: 550-557, 2008.

48. Li J and Zhang S: The effect of mild hypothermia on mice cerebral ischemia-reperfusion nerve cell apoptosis. Chin J Neurosurg 26: 904-907, 2010 (In Chinese).

49. Ye X, Yu S, Li C and Guo L: Neuroprotective role of mild hypothermia on cerebral ischemia-reperfusion injury in rats. Clin Med China 22: 124-126, 2006 (In Chinese).

50. Xue BS, Feng PH, Wei ND, Li ZF, Li YY, Lv XL and Hou WJ The effect and mechanism of hypothermia on repair of cerebra ischemia-reperfusion injury rats. Prog Anat Sci 22: 654-657, 2016 (In Chinese)

51. Cao Z, Balasubramanian A, Pedersen SE, Romero J, Pautler RG and Marrelli SP: TRPV1-mediated pharmacological hypothermia promotes improved functional recovery following ischemic stroke. Sci Rep 7: 17685, 2017.

52. Lay $\mathrm{C}$ and Badjatia N: Therapeutic hypothermia after cardiac arrest. Curr Atheroscler Rep 12: 336-342, 2010.

53. Ai L, Qiao Q, Chen N, Yang T, Tang X and Yue J: Neuroprotective effect of therapeutic hypothermia induced by dihydrocapsaicin on cerebral ischemia reperfusion injury in mice. J Xinxiang Med Univ 34: 1058-1062, 2017 (In Chinese).

54. Muzzi M, Felici R, Cavone L, Gerace E, Minassi A, Appendino G, Moroni $\mathrm{F}$ and Chiarugi A: Ischemic neuroprotection by TRPV1 receptor-induced hypothermia. J Cereb Blood Flow Metab 32: 978-982, 2012

55. Cao Z, Balasubramanian A and Marrelli SP: Pharmacologically induced hypothermia via TRPV1 channel agonism provides neuroprotection following ischemic stroke when initiated $90 \mathrm{~min}$ after reperfusion. Am J Physiol Regul Integr Comp Physiol 306 R149-R156, 2014.

56. Shibasaki K: Physiological significance of TRPV2 as a mechanosensor, thermosensor and lipid sensor. J Physiol Sci 66 : $359-365,2016$

57. Shibasaki K, Ishizaki Y and Mandadi S: Astrocytes express functional TRPV2 ion channels. Biochem Biophys Res Commun 441: 327-332, 2013

58. Deng Q: Efficacy evaluation of Erigeron breviscapus on neurological function recovery after minimally invasive procedures for removal of intracranial hematoma. Chin J Pract Nerv Dis 12 : 82-84, 2009

59. Kojima I and Nagasawa M: TRPV2. Handb Exp Pharmacol 222 247-272, 2014.

60. Park HJ, Kwon H, Lee S, Jung JW, Ryu JH, Jang DS, Lee YC and Kim DH: Echinocystic acid facilitates neurite outgrowth in neuroblastoma Neuro2a cells and enhances spatial memory in aged mice. Biol Pharm Bull 40: 1724-1729, 2017

61. Cohen MR, Johnson WM, Pilat JM, Kiselar J, DeFrancescoLisowitz A, Zigmond RE and Moiseenkova-Bell VY: Nerve growth factor regulates transient receptor potential vanilloid 2 via extracellular signal-regulated kinase signaling to enhance neurite outgrowth in developing neurons. Mol Cell Biol 35: 4238-4252, 2015.
62. Zhang H, Xiao J, Hu Z, Xie M, Wang W and He D: Blocking transient receptor potential vanilloid 2 channel in astrocytes enhances astrocyte-mediated neuroprotection after oxygen-glucose deprivation and reoxygenation. Eur J Neurosci 44: 2493-2503, 2016.

63. Xiao J, Yang F, Zhang H, Wang W and He D: TRPV2 activation enhances the expression of nerve growth factor in primary cultured astrocytes under oxygen-glucose deprivation/reoxygenation. Chin J Cell Biol 36: 773-779, 2014.

64. Luo H, Rossi E, Saubamea B, Chasseigneaux S, Cochois V, Choublier N, Smirnova M, Glacial F, Perrière N, Bourdoulous S, et al: Cannabidiol increases proliferation, migration, tubulogenesis, and integrity of human brain endothelial cells through TRPV2 activation. Mol Pharm 16: 1312-1326, 2019.

65. Garcia-Elias A, Mrkonjic S, Jung C, Pardo-Pastor C, Vicente R and Valverde MA: The TRPV4 channel. Handb Exp Pharmacol 222: 293-319, 2014.

66. Nilius B and Voets T: The puzzle of TRPV4 channelopathies. EMBO Rep 14: 152-163, 2013.

67. Liedtke W, Choe Y, Martí-Renom MA, Bell AM, Denis CS Sali A, Hudspeth AJ, Friedman JM and Heller S: Vanilloid receptor-related osmotically activated channel (VR-OAC), a candidate vertebrate osmoreceptor. Cell 103: 525-535, 2000.

68. Li L, Liu C, Chen L and Chen L: Hypotonicity modulates tetrodotoxin-sensitive sodium current in trigeminal ganglion neurons. Mol Pain 7: 27, 2011

69. Chen NN, Wang JP, Jiang C, Liu C, Li X, Zhao Y and Hao Y: Research about the influence of progesterone on expression of COX-2 and the water content of injured brain in cerebral ischemia in rats. J Apoplexy Nerv Dis 6: 671-673, 2009.

70. Lu WC, Ma YJ, Xie H, Dig XH, Su QX, Xing HH, Meng YH, Fan J and Tian JH: Effect of TRPV4 channel on focal cerebral ischemic reperfusion injury in rats. Prog Anat Sci 23: 353-355, 2017 (In Chinese)

71. Lipski J, Park TI, Li D, Lee SC, Trevarton AJ, Chung KK, Freestone PS and Bai JZ: Involvement of TRP-like channels in the acute ischemic response of hippocampal CA1 neurons in brain slices. Brain Res 1077: 187-199, 2006.

72. Zacharia BE, Hickman ZL, Grobelny BT, DeRosa PA, Ducruet AF and Connolly ES: Complement inhibition as a proposed neuroprotective strategy following cardiac arrest. Mediators Inflamm 2009: 124384, 2009.

73. Bernard SA, Gray TW, Buist MD, Jones BM, Silvester W, Gutteridge G and Smith K: Treatment of comatose survivors of out-of-hospital cardiac arrest with induced hypothermia. N Engl J Med 346: 557-563, 2002.

74. Wu Q, Qian C, Zhao N, Dong Q, Li J, Wang BB, Chen L, Yu L, Han B, Du YM and Liao YH: Activation of transient receptor potential vanilloid 4 involves in hypoxia/reoxygenation injury in cardiomyocytes. Cell Death Dis 8: e2828, 2017

75. Jie P, Hong Z, Tian Y, Li Y, Lin L, Zhou L, Du Y, Chen L and Chen L: Activation of transient receptor potential vanilloid 4 induces apoptosis in hippocampus through downregulating PI3K/Akt and upregulating p38 MAPK signaling pathways. Cell Death Dis 6: e1775, 2015

76. Jie P, Lu Z, Hong Z, Li L, Zhou L, Li Y, Zhou R, Zhou Y, Du Y, Chen $L$ and Chen L: Activation of transient receptor potential vanilloid 4 is involved in neuronal injury in middle cerebral artery occlusion in mice. Mol Neurobiol 53: 8-17, 2016.

77. Butenko O, Dzamba D, Benesova J, Honsa P, Benfenati V, Rusnakova V, Ferroni S and Anderova M: The increased activity of TRPV4 channel in the astrocytes of the adult rat hippocampus after cerebral hypoxia/ischemia. PLoS One 7: e39959, 2012.

78. Dunn KM, Hill-Eubanks DC, Liedtke WB and Nelson MT: TRPV4 channels stimulate $\mathrm{Ca} 2+$-induced $\mathrm{Ca} 2+$ release in astrocytic endfeet and amplify neurovascular coupling responses. Proc Natl Acad Sci USA 110: 6157-6162, 2013

79. Zhang YF, Fan XJ, Li X, Peng LL, Wang GH, Ke KF and Jiang ZL: Ginsenoside Rg1 protects neurons from hypoxic-ischemic injury possibly by inhibiting $\mathrm{Ca} 2+$ influx through NMDA receptors and L-type voltage-dependent Ca2+ channels. Eur J Pharmacol 586 90-99, 2008

80. Tanaka K, Matsumoto S, Yamada T, Yamasaki R, Suzuki M, Kido MA and Kira JI: Reduced post-ischemic brain injury in transient receptor potential vanilloid 4 knockout mice. Front Neurosci 14: 453, 2020.

81. Diaz-Otero JM, Yen TC, Ahmad A, Laimon-Thomson E, Abolibdeh B, Kelly K, Lewis MT, Wiseman RW, Jackson WF and Dorrance AM: Transient receptor potential vanilloid 4 channels are important regulators of parenchymal arteriole dilation and cognitive function. Microcirculation 26: e12535, 2019. 
82. Simpson S, Preston D, Schwerk C, Schroten H and Blazer-Yost B Cytokine and inflammatory mediator effects on TRPV4 function in choroid plexus epithelial cells. Am J Physiol Cell Physiol 317: C881-C893, 2019

83. Emsley HC and Tyrrell PJ: Inflammation and infection in clinical stroke. J Cereb Blood Flow Metab 22: 1399-1419, 2002.

84.Zhu D, Y in L and Liu Q: The role of TRPV4 in OGD damage of cultured in vitro astrocytes. Chin J Clin Res 26: 885-888, 2013.

85. Hong Z, Tian Y, Qi M, Li Y, Du Y, Chen L, Liu W and Chen L: Transient receptor potential vanilloid 4 inhibits $\gamma$-aminobutyric acid-activated current in hippocampal pyramidal neurons. Front Mol Neurosci 9: 77, 2016.

86. Ashraf MI, Ebner M, Wallner C, Haller M, Khalid S, Schwelberger H, Koziel K, Enthammer M, Hermann M, Sickinger S, et al: A p38MAPK/MK2 signaling pathway leading to redox stress, cell death and ischemia/reperfusion injury. Cell Commun Signal 12: 6, 2014.

87. Morente V,Pérez-Sen R, Ortega F, Huerta-Cepas J, Delicado EG and Miras-Portugal MT: Neuroprotection elicited by P2Y13 receptors against genotoxic stress by inducing DUSP2 expression and MAPK signaling recovery. Biochim Biophys Acta 1843: 1886-1898, 2014.

88. Maddahi A, Chen Q and Edvinsson L: Enhanced cerebrovascular expression of matrix metalloproteinase- 9 and tissue inhibitor of metalloproteinase-1 via the MEK/ERK pathway during cerebral ischemia in the rat. Bmc Neurosci 10: 56, 2009.

89. Kovalska M, Kovalska L, Pavlikova M, Janickova M, Mikuskova K, Adamkov M, Kaplan P, Tatarkova Z and Lehotsky J: Intracellular signaling MAPK pathway after cerebral ischemia-reperfusion injury. Neurochem Res 37: 1568-1577, 2012.

90. Rumbaut RE, McKay MK and Huxley VH: Capillary hydraulic conductivity is decreased by nitric oxide synthase inhibition. Am J Physiol 268: H1856-H1861, 1995.

91. Piao CS, Kim JB, Han PL and Lee JK: Administration of the p38 MAPK inhibitor SB203580 affords brain protection with a wide therapeutic window against focal ischemic insult. J Neurosci Res 73: 537-544, 2003.

92. Kang J, Zhang Y, Cao X, Fan J, Li G, Wang Q, Diao Y, Zhao Z, Luo L and Yin Z: Lycorine inhibits lipopolysaccharide-induced iNOS and COX-2 up-regulation in RAW264.7 cells through suppressing P38 and STATs activation and increases the survival rate of mice after LPS challenge. Int Immunopharmacol 12: 249-256, 2012.

93. Ridnour LA, Windhausen AN, Isenberg JS, Yeung N, Thomas DD, Vitek MP, Roberts DD and Wink DA: Nitric oxide regulates matrix metalloproteinase- 9 activity by guanylyl-cyclase-dependent and -independent pathways. Proc Natl Acad Sci USA 104: 16898-16903, 2007.
94. Okuno S, Saito A, Hayashi T and Chan PH: The c-Jun N-terminal protein kinase signaling pathway mediates Bax activation and subsequent neuronal apoptosis through interaction with Bim after transient focal cerebral ischemia. J Neurosci 24: 7879-7887, 2004.

95. Gao X, Zhang H, Takahashi T, Hsieh J, Liao J, Steinberg GK and Zhao $\mathrm{H}$ : The Akt signaling pathway contributes to postconditioning's protection against stroke; the protection is associated with the MAPK and PKC pathways. J Neurochem 105: 943-955, 2008.

96. Sun T, Li YJ, Tian QQ, Wu Q, Feng D, Xue Z, Guo YY, Yang L, Zhang K, Zhao MG and Wu YM: Activation of liver X receptor $\beta$-enhancing neurogenesis ameliorates cognitive impairment induced by chronic cerebral hypoperfusion. Exp Neurol 304: 21-29, 2018.

97. Weiss HR, Chi OZ, Kiss GK, Liu X, Damito S and Jacinto E: Akt activation improves microregional oxygen supply/consumption balance after cerebral ischemia-reperfusion. Brain Res 1683: 48-54, 2018

98. Gao F, Gao E, Yue TL, Ohlstein EH, Lopez BL, Christopher TA and Ma XL: Nitric oxide mediates the antiapoptotic effect of insulin in myocardial ischemia-reperfusion: The roles of PI3-kinase, Akt, and endothelial nitric oxide synthase phosphorylation. Circulation 105: 1497-1502, 2002.

99. Rocha-Ferreira E, Rudge B, Hughes MP, Rahim AA, Hristova M and Robertson NJ: Immediate remote ischemic postconditioning reduces brain nitrotyrosine formation in a piglet asphyxia model. Oxid Med Cell Longev 2016: 5763743, 2016.

100. Kamada H, Nito C, Endo $\mathrm{H}$ and Chan PH: Bad as a converging signaling molecule between survival PI3-K/Akt and death JNK in neurons after transient focal cerebral ischemia in rats. J Cereb Blood Flow Metab 27: 521-533, 2007.

101. Li F, Omori N, Jin G, Wang SJ, Sato K, Nagano I, Shoji M and Abe K: Cooperative expression of survival p-ERK and p-Akt signals in rat brain neurons after transient MCAO. Brain Res 962: 21-26, 2003

102. Berliocchi L, Bano D and Nicotera P: Ca2+ signals and death programmes in neurons. Philos Trans R Soc Lond B Biol Sci 360: 2255-2258, 2005 .

103. Zhang E and Liao P: Brain transient receptor potential channels and stroke. J Neurosci Res 93: 1165-1183, 2015.

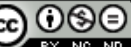

This work is licensed under a Creative Commons Attribution-NonCommercial-NoDerivatives 4.0 International (CC BY-NC-ND 4.0) License. 